\title{
Efecto del área disponible entre colocación y primer tiempo en voleibol masculino de élite
}

\author{
Effect of the available area between setter and middle-attacker in \\ elite men volleyball.
}

\section{Autora: Elia Mercado Palomino}

Director: Aurelio Ureña Espá (Departamento de Actividad Física y Deportiva) Co-Director: José Manuel Benítez Sánchez (Ciencias de la Computación e Inteligencia Artificial)
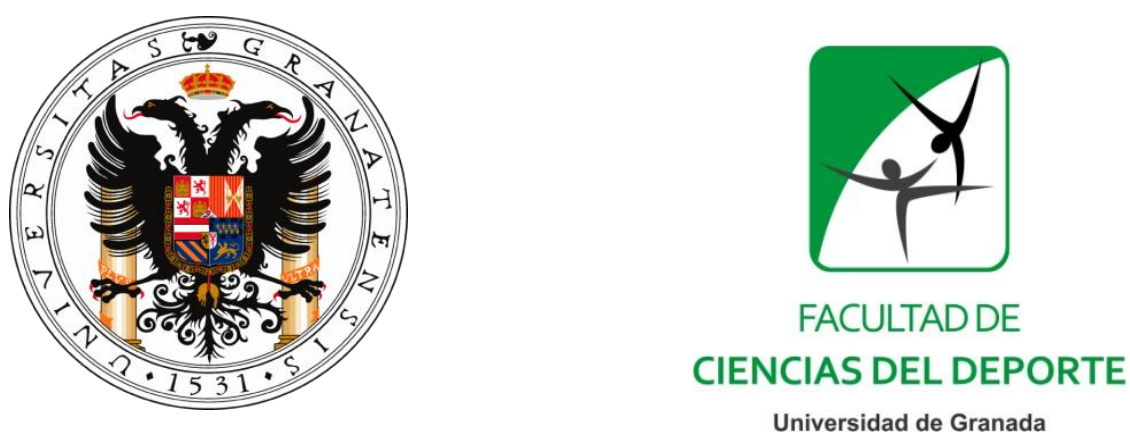

Máster de Investigación en Actividad Física y Deporte Trabajo Fin de Máster

Departamento de Actividad física y Deportiva

Facultad de Ciencias del Deporte

Universidad de Granada 


\section{Índice}

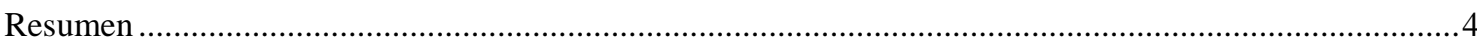

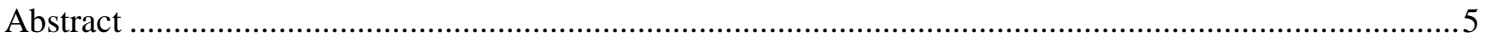

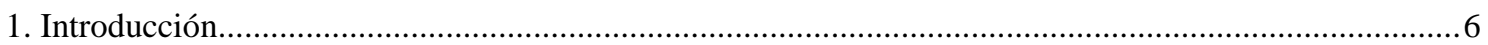

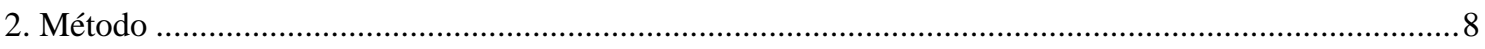

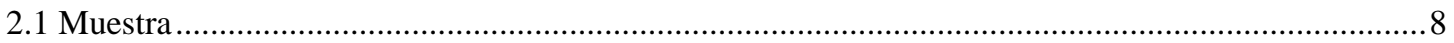

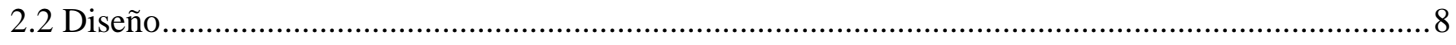

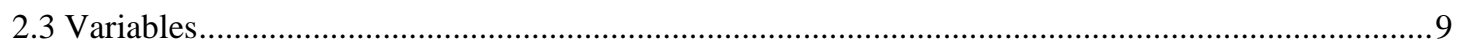

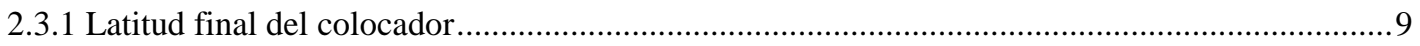

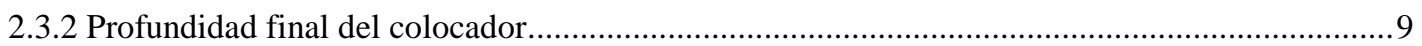

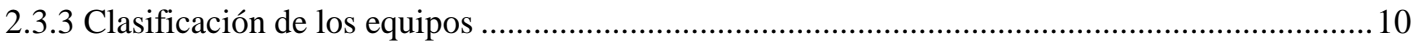

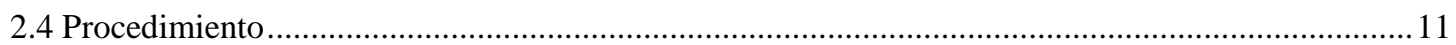

2.4.1 Criterios de registro para la Latitud y Profundidad final del colocador ................................... 11

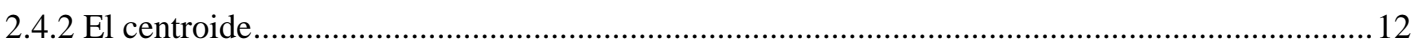

2.4.3 Cálculo del vector con respecto al centro de densidad ...................................................... 13

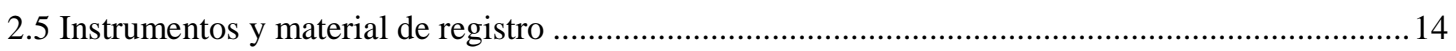

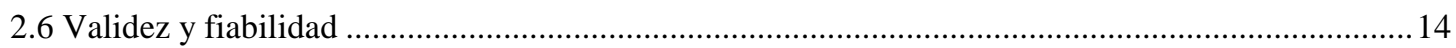

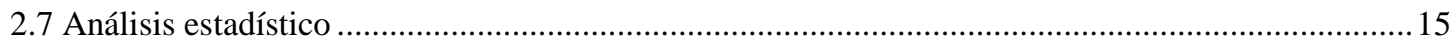

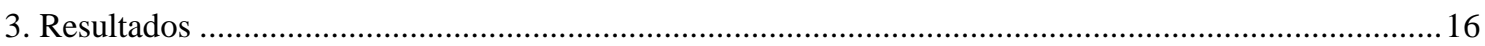

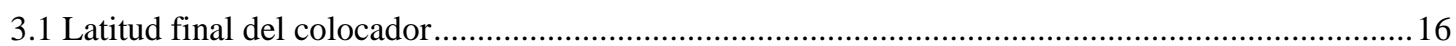

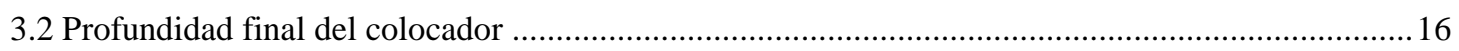

3.3 Frecuencias de todos los equipos de la posición final del colocador .............................................. 17

3.4 Vector, ángulo y centroide de cada equipo ............................................................................ 18

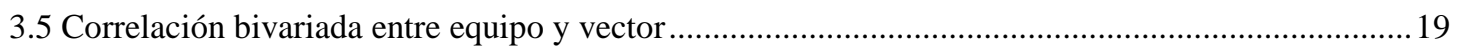

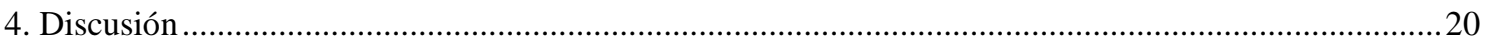

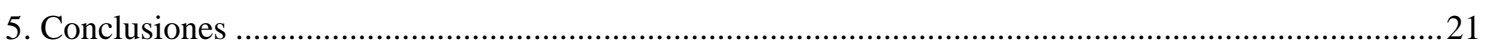

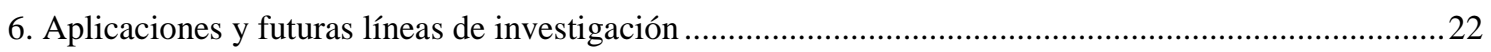

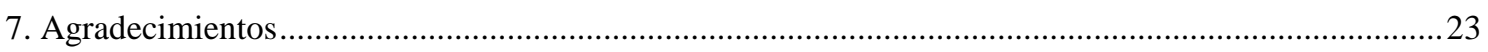

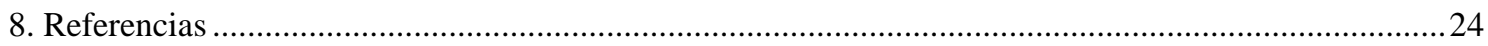




\section{Resumen}

Con el presente estudio, se pretendió medir las variaciones en la disponibilidad del primer tiempo de los equipos, a partir de la distribución de frecuencias de colocación a lo largo de la superficie de juego en las que el equipo mantiene esa disponibilidad; encontrar un valor resumen que permita la comparación entre equipos y su relación con otras variables; y detectar si esa medida es un indicador de rendimiento, evaluando la relación que tiene ese valor en el puesto de cada equipo en la clasificación final. Se analizó el Campeonato del Mundo de Voleibol Masculino de 2010 en Italia, con un total de 23 partidos y representada por 12 nacionalidades, utilizando las variables Latitud y Profundidad final del colocador, y calculando el centroide, el vector y el ángulo de cada uno de los equipos. Los resultados sugieren que existe relación entre el área de disponibilidad del primer tiempo y la clasificación de los equipos $(p<0.01)$, de manera que, cuanto mayor sea el área disponible, mayor será la disponibilidad de ataque de primer tiempo, y por tanto, mayor será el rendimiento de un equipo $\left(r_{s}=0.783\right)$, cuestionando la calidad de la recepción, medida desde la precisión, como un indicador suficiente. En conclusión, el centroide es un valor resumen del área de disponibilidad del primer tiempo, que nos permite comparar unos equipos con otros, a partir de la distribución de frecuencias de colocación, y al mismo tiempo, es un indicador de rendimiento, en relación con el puesto de cada equipo en la clasificación final.

Palabras clave: análisis del juego, voleibol, colocador, primer tiempo, centroide, ataque. 


\section{Abstract}

The present study intended to measure the changes in the availability of the middleattacker of volleyball teams, from the setting frequencies distribution throughout the court, where the team maintains that availability; finding a summary value that allows the comparison between teams and their relationship with other variables; and detecting whether the measure is a performance indicator, evaluating the relationship of this value with the position of each team in the final standings. Volleyball Men's World Championship 2010 in Italy was analyzed, with a total of 23 games and represented by 12 national teams, using the variables Latitude and Depth of the setter, and calculating the centroid, the vector and the angle for each team. The results suggest that a relationship exists between the availability of the middle-attacker and final ranking of teams $(p<0.01)$, so that, the bigger the available area, the more availability the middle-attacker has, and therefore the greater the performance of the team $\left(r_{s}=0.783\right)$, questioning the reception quality measured from the accuracy, as an adequate indicator. In conclusion, the centroid is a summary value of the area of availability of the middle-attacker, allowing us to make comparisons between teams, from the setting frequencies distribution, and at the same time, it is a performance indicator in relation to the position of each team in the final ranking.

Keywords: match analysis, volleyball, setter, first tempo attack, centroid, attack. 


\section{Introducción}

El voleibol, debido a su naturaleza compleja, dinámica y no lineal (Davids et al., 2003), es un deporte muy rico en componentes técnicos y tácticos, que conllevan una fuerte relación entre factores que ocurren primero y los posteriores. Su estudio a través del análisis de los indicadores de rendimiento es fundamental para cuantificar los elementos clave de una manera válida y consistente (ISPAS, 2015). Del mismo modo, se hace necesario este tipo de análisis para mejorar los procesos de entrenamiento y favorecer el desarrollo de estrategias que estimulen la mejora del rendimiento de los equipos (Afonso y Mesquita, 2011; Garganta, 2009; Jager y Schollhorn, 2007).

En voleibol, el colocador es el responsable del segundo contacto, y por tanto de preparar el balón para que los atacantes culminen con éxito (Afonso et al., 2010; Nikos y Elissavet, 2011). Posee una clara responsabilidad organizativa en las habilidades ofensivas del equipo (Castro y Mesquita, 2008; Zetou et al., 2007). Así pues, el colocador es una pieza clave para el éxito de cualquier equipo de voleibol debido a su relación directa con el éxito del equipo (Buscà y Febrer, 2012; Morales, 2011; Palao et al., 2005; Papadimitriou et al., 2004; Ureña, 1998).

Durante el juego, se considera que un equipo se encuentra en Complejo I (K1) cuando el equipo que recibe el saque, ejecuta las acciones de recepción, colocación y ataque de forma secuencial (Bertucci, 1979). La investigación hasta el momento se ha encargado de reforzar la influencia que tiene la precisión de la recepción en la eficacia (Nikos et al., 2011; Zetou et al., 2007). Sin embargo, la disponibilidad del atacante central para ataques rápidos (entendiéndose como aquellos ataques de primer tiempo, en los cuales el atacante salta durante la colocación o justo después, posiblemente dando un paso después de la colocación) es necesaria para que el equipo tenga una rápida y diversificada ofensiva (Afonso et al., 2010). En K1 el rendimiento de los atacantes es altamente dependiente del rendimiento del colocador, de forma que la eficacia excelente del ataque pasa de un $51,4 \%$ a un $79,4 \%$ en función de si es una colocación buena o perfecta, respectivamente (Barzouka et al. 2006). Además, generar ataques rápidos desde la colocación permite crear situaciones ofensivas con desequilibrio en la cohesión del bloqueo contrario (Afonso et al., 2010), permitiendo así al atacante igualdad (un bloqueo) o superioridad al no tener ningún bloqueo (Queiroga et al., 2005). 
Esto ha llevado a valorar la calidad de la recepción de forma universal, en función de la zona y la acción técnica. Sin embargo, la capacidad que tiene un colocador en la relación con su atacante de primer tiempo ces universal y depende linealmente de la precisión de la recepción? o ¿̇se pueden establecer radios de acción que puedan variar significativamente de unos equipos a otros? Desde esa inquietud, en lugar de evaluar la calidad de la recepción siguiendo los criterios ya validados en distintos estudios (Castro et al., 2011; Costa et al., 2011; Zetou et al., 2007), nos hemos planteado discriminar a los equipos en la medida de la disponibilidad espacial del primer tiempo de ataque. Del mismo modo, queremos establecer si la medida de esa disponibilidad puede considerarse un indicador de rendimiento.

En la línea de lo propuesto, se realizó la búsqueda de una unidad que pudiese resumir la distribución de frecuencias en un punto, se encontró el término centroide como un algoritmo de promediado estadístico. Dentro del área de ciencias del deporte se viene utilizando convencionalmente en cinemática para calcular el centro de masas de los segmentos corporales (Mapelli et al., 2014). Recientemente se ha encontrado una aplicación en análisis de la táctica como variable para determinar la distribución de los equipos, ya sea entre equipos o entre sus jugadores (Folgado et al., 2014; Frencken et al., 2011).

Así pues, los objetivos de este estudio son: 1) medir las variaciones en la disponibilidad del primer tiempo de los equipos a partir de la distribución de frecuencias de colocación a lo largo de la superficie de juego en las que el equipo mantiene esa disponibilidad, 2) encontrar un valor resumen que permita la comparación entre equipos y su relación con otras variables, y 3) detectar si esa medida es un indicador de rendimiento, evaluando la relación que tiene ese valor en el puesto de cada equipo en la clasificación final. 


\section{Método}

\subsection{Muestra}

Se analizó el Campeonato del Mundo de voleibol masculino de 2010 en Italia de la Federación Internacional de Voleibol (FIVB), con un total de 23 partidos y sus correspondientes 87 sets. La muestra fue representada por 12 nacionalidades: Alemania, Argentina, Brasil, Bulgaria, Cuba, España, Francia, Italia, República Checa, Rusia, Serbia y EEUU.

Para asegurar la competitividad y el alto nivel de los participantes, todos los partidos seleccionados se corresponden con la fase final del campeonato, la cual estuvo compuesta por un total de 78 partidos, correspondiente a un $29.48 \%$ del total de los partidos. En dicha fase los equipos con menos partidos jugados acumularon 3 partidos y los finalistas hasta 5 . Se seleccionaron aleatoriamente para cada selección entre 3 y 4 partidos.

Las variables fueron registradas únicamente en aquellas jugadas en las que el central del equipo en $\mathrm{K} 1$, se mostraba eficazmente disponible para ejecutar un ataque rápido, ya sea simulando un ataque rápido por delante (cerca o lejos) o detrás del colocador, cumpliendo los criterios de Alfonso et al., (2010).

\subsection{Diseño}

Se trata de un estudio descriptivo de metodología observacional, ya que tiene un grado de control interno bajo para no desnaturalizar el juego. Sin embargo, cuenta con un grado de perceptividad máximo, ya que se ha realizado mediante observación directa, a través de análisis de vídeos.

Es un diseño de seguimiento, nomotético y multidimensional, ya que analizamos diversos partidos con el fin de generalizar los resultados a la población estudiada mediante el registro de las conductas de diferentes jugadores y equipos, y teniendo en cuenta la multitud de variables a analizar debido a la complejidad y a la alta incertidumbre de este deporte. 


\subsection{Variables}

\subsubsection{Latitud final del colocador}

El campo de voleibol tiene 9 metros de anchura, se dividió la red en 9 columnas diferentes de un metro cada una, obteniendo así la latitud de la pista (Figura 1).

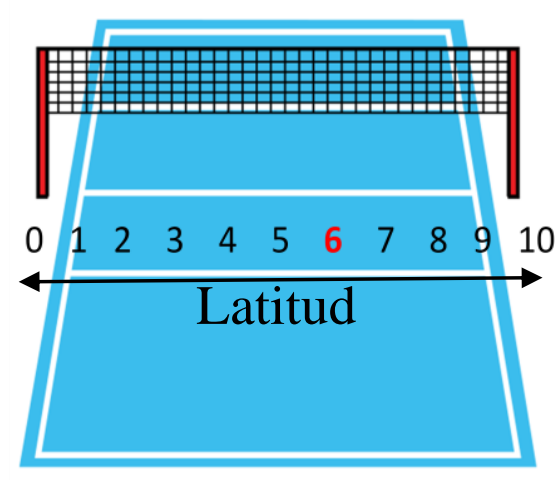

Figura 1. Latitud del campo de voleibol

Para que se cumpla la mutua exclusividad, cuando el colocador se situaba fuera de pista por el lado derecho, se registró como zona de colocación 0 , y cuando era por el lado izquierdo se registró como zona de colocación 10.

Para asegurar rigor, se estableció que en situación de duda a la hora de registrar una zona, siempre se escogerá aquella más cercana a la zona 6 , ya que es establecida como la zona ideal de colocación partiendo de lo propuesto por Beal (1992).

\subsubsection{Profundidad final del colocador}

Para registrar la profundidad, se dividió el campo de voleibol en 9 filas de 1 metro cada una, desde la 1 que es la más cercana a la red, hasta la 9 que es la más alejada (Figura 2).

Para asegurar rigor en el registro, se estableció que en situación de duda a la hora de registrar una zona, se escogió aquella más cercana a la zona 1, ya que es establecida como profundidad ideal de colocación. 


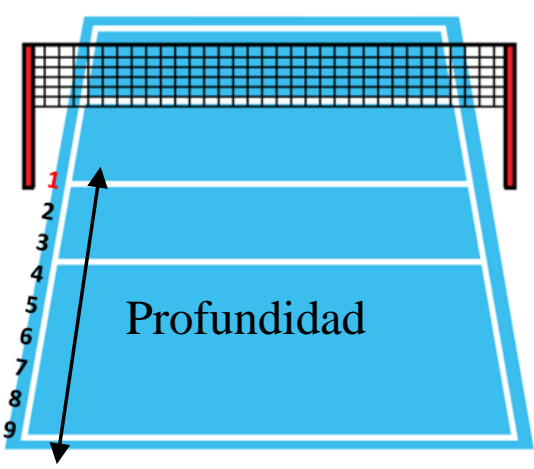

Figura 2. Profundidad del campo de voleibol

De esta forma queda el campo de voleibol estructurado en una cuadrícula, en la cual, cada área tiene unas coordenadas para la localización precisa del colocador (Figura 3).

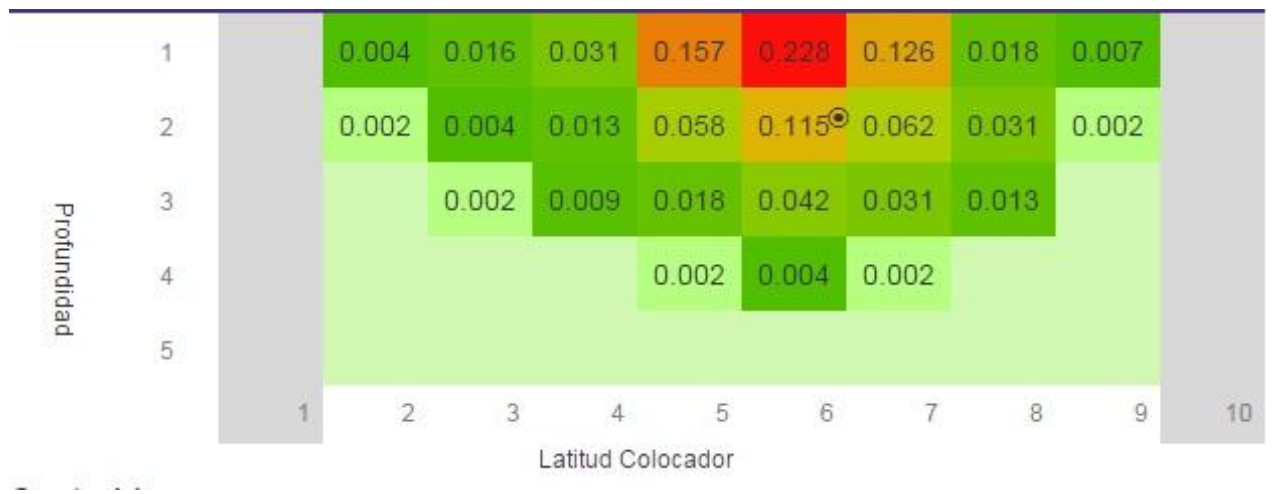

Figura 3. Representación del campo de voleibol de las frecuencias relativas del colocador de España en el campeonato del Mundo de 2010 de Voleibol Masculino.

\subsubsection{Clasificación de los equipos}

Se utilizó la clasificación final de los equipos del Campeonato del Mundo 2010 como variable de rendimiento en función del ranking de éstos (Figura 4).

$\begin{array}{cl}\text { Rank } & \text { Country } \\ \text { 1st } & \text { Brazil } \\ \text { 2nd } \square \text { Cuba } \\ \text { 3rd } \square \text { Serbia } \\ \text { 4th } \square \text { Italy } \\ \text { 5th } \square \text { Russia } \\ \text { 6th } \square \text { USA } \\ \text { 7th } \square \text { Bulgaria } \\ \text { 8th } \square \text { Germany } \\ \text { 9th } \square \text { Argentina } \\ \text { 10th } \square \text { Czech Republic } \\ \text { 11th } \square \text { France } \\ \text { 12th } \square \text { Spain }\end{array}$

Figura 4. Clasificación final de los equipos del campeonato del Mundo de 2010 de Voleibol Masculino (FIVB) 


\subsection{Procedimiento}

\subsubsection{Criterios de registro para la Latitud y Profundidad final del colocador}

- Momento de registro: el momento en que se registra la zona dónde se ejecuta la colocación es aquella en la que se ejecutó el último apoyo antes de realizar la colocación o el salto para la colocación.

\section{- Situaciones:}

- Cuando se trata de una colocación en salto: se registra siempre la zona del último/últimos apoyo/s antes de realizar el salto para la colocación. Se pueden dar dos situaciones:

$>$ Que el colocador tenga ambos pies en apoyo justo antes del salto. En dicho caso se sigue el criterio principal de zona 16, es decir se elige el pie más cercano a dicha zona. Si dicho pie se ubica entre dos zonas, se seguirá de nuevo el mismo criterio de zona 16.

Que el colocador tenga un solo pie en apoyo justo antes del salto. En este caso se escoge dicho pie, y si éste está situado entre dos celdas se seguirá de nuevo el criterio de zona 16.

- Cuando se trata de una colocación sin salto: se registra siempre la zona del último/últimos apoyo/s antes de realizar la colocación (gesto técnico de pase de dedos). Se puede dar que el colocador tenga un pie o ambos apoyados, por lo que se siguió el criterio anterior.

\section{- Nombre y código de las variables en la base de datos:}

- Latitud final del colocador: LAT_COL

- Profundidad final del colocador: PRO_COL

La grabación de los partidos se realizó desde una posición superior a la pista, con perfecta visión de la totalidad de ésta, sin modificación de su posición o imagen y sin cortes durante la filmación, evitando así errores de procedimiento. 


\subsubsection{El centroide}

El centroide es una operación matemática y geométrica, que permite conocer la posición dentro de la cuadrícula donde está la media ponderada de filas y columnas de todo el campo. Se usa la media ponderada, debido a que cada casilla del campo, contiene un valor de frecuencia que asocia cada sector (cuadrante) del campo a un peso concreto, de modo que para todas las filas y las columnas separadamente se obtiene un valor ponderado, que luego se promedia para obtener las coordenadas $(x, y)$ del centroide.

A partir de la cuadrícula de datos de cada equipo, cada celda tiene una frecuencia relativa correspondiente al porcentaje de jugadas que realiza en cada posición (número de jugadas en cada posición / total de jugadas), mostrando las zonas donde se concentra más el colocador. Los colores representan la escala de frecuencias de colocación, de manera que el rojo es el valor más alto y se va degradando hasta el verde oscuro (Figura 5).

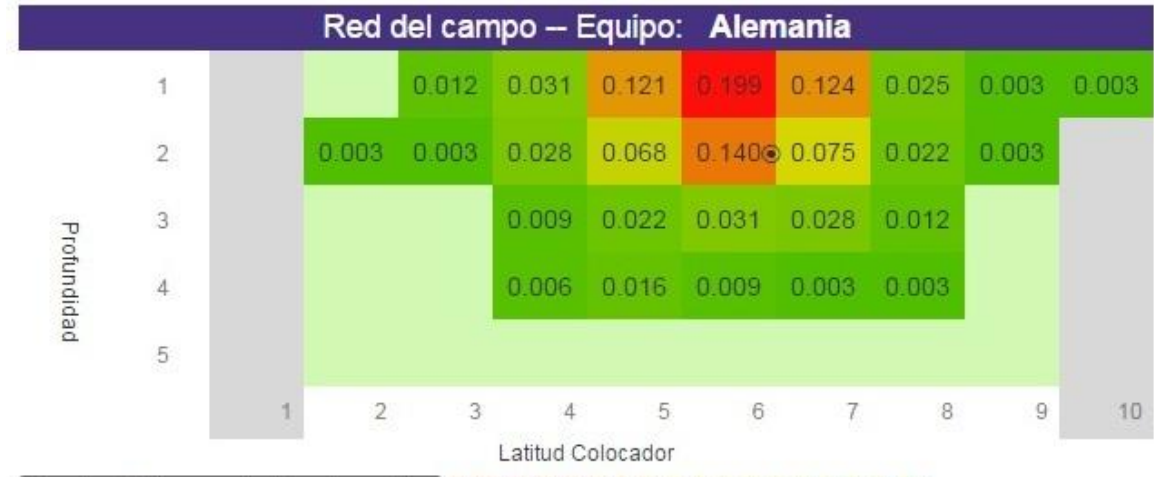

Escala de frecuencia de colocación 80 |||||||||||||||||||||||||||||||||||||||||||||||||||| 1

Figura 5. Representación del centroide de Alemania en el campeonato del Mundo de 2010 de Voleibol Masculino.

Para calcular el centroide (Figura 6), se calculó el valor de cada fila, multiplicando el valor de cada celda por su frecuencia, y posteriormente se sumaron todos los datos de esa fila, obteniendo $f 1, f 2, f 3, f 4, f 5 \ldots$

\section{$\begin{array}{lllllllll}0.012 & 0.031 & 0.121 & 0.199 & 0.124 & 0.025 & 0.003 & 0.003\end{array}$}

Figura 6: Ejemplo de cálculo de $f 1: 1 * 0+2 * 0+3 * 0.012+4 * 0.031+5 * 0.121+\ldots+10 * 0.003$ 
Con los valores obtenidos de las filas, se calculó la media, dándonos así el valor medio de la profundidad del centroide. Para obtener la latitud del centroide se realizó el mismo proceso, pero con las columnas.

Finalmente, se obtuvieron unas coordenadas de la profundidad y latitud del centroide. En este caso, como podemos ver en la Figura 5, se corresponde con $(5.946,1.656)$.

\subsubsection{Cálculo del vector con respecto al centro de densidad}

El centro de densidad se corresponde en la cuadrícula con el punto céntrico de la celda de latitud 6 y profundidad 1 , con coordenadas $(5.5,0.5)$, la cual es la zona de mayor frecuencia en todos los equipos.

El vector es la distancia existente entre el centro de densidad con respecto al centroide, y se calculó del siguiente modo (Figura 7):

- Centro de densidad: $(5.5,0.5)$

- Centroide: $\quad$ (Latitud del centroide, Profundidad del centroide)

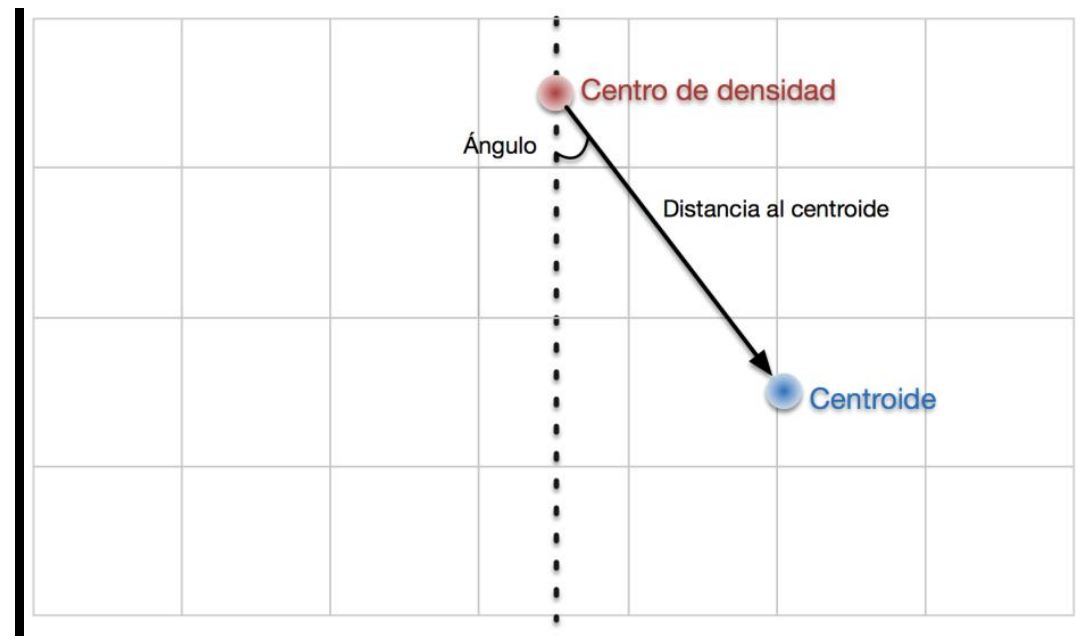

Figura 7. Representación del cálculo del vector y el ángulo con respecto al centro de densidad.

Si consideramos el centro de densidad como $P\left(x_{p}, y_{p}\right)$, y el centroide como $C\left(x_{c}, y_{c}\right)$, la distancia del vector se calcularía con la siguiente fórmula:

$$
\sqrt[2]{|x c-x p|^{2}+|y p-y c|^{2}}
$$


El ángulo en radianes se calculó con la siguiente fórmula $\left(\operatorname{arctg} \frac{y c-y p}{x c-x p}\right) \mathrm{y}$ posteriormente se pasó a grados multiplicando el resultado en radianes por 180 y dividiéndolo por $\pi$.

Para calcular el centroide medio de todos los equipos, se realizó el cálculo del centroide y vector de cada uno, y posteriormente la media de todos ellos.

\subsection{Instrumentos y material de registro}

Para el análisis observacional se utilizó el software VA-Sport (versión 1.0.74, Desarrollo Software Deportivo, España) desarrollado en el marco del Proyecto de Investigación "Sistema MasVb de Evaluación Competitiva y Orientación Técnica para la Superliga Española de Voleibol" (DEP2011-27503), financiado por el Ministerio de Ciencia e Innovación dirigido por la Universidad de Granada.

En el proyecto, se desarrolló para el software una herramienta que ha sido aprovechada para este trabajo, que permite incorporar al vídeo una rejilla, delimitando cada una de las zonas del campo, y que es perfectamente ajustable a la pista de voleibol, adaptándose independientemente del ángulo de la cámara y de su orientación. Esto supone un avance en la precisión espacial que ha sido una limitación habitual en los estudios mediante técnicas de observación.

\subsection{Validez y fiabilidad}

Para determinar la fiabilidad del sistema de categorías se llevó a cabo un análisis intra e inter-observador de todas las variables registradas, de forma que se recogió un 10\% aleatorio de la muestra (Tabachnick y Fidell, 2011). Como podemos ver en la Tabla 1, los valores de Kappa de Cohen (1960) de todos los análisis fueron mayores de 0.75 tomando como referencia los valores propuestos por Fleiss (2003).

Tabla 1. Fiabilidad Intra-observador e inter-observador

\begin{tabular}{llll}
\hline Cohen's Kappa & Intra-observador & Inter-observador & N (aleatorio) \\
Situación K1 perfecta & 0.942 & 0.948 & 394 \\
Latitud final del colocador & 0.870 & 0.858 & 235 \\
Profundidad final del colocador & 0.801 & 0.784 & 235 \\
& & & \\
\hline
\end{tabular}


Para controlar la expectancia, el registro fue hecho por un observador entrenado que no conocía el objetivo del estudio.

Por otro lado, tampoco existe sesgo por reactividad, debido a la costumbre de los jugadores de élite de ser grabados. Además, como los partidos son grabados previos a los estudios, no conocen el objetivo de estos y actúan de forma espontánea.

El proyecto ha sido aprobado por el Comité de Ética en Investigación Humana de la Universidad de Granada (adjunto en el CD).

\subsection{Análisis estadístico}

Para el tratamiento descriptivo de los datos se empleó un estudio de frecuencias y porcentajes, a través de los cuales se calculó el centroide y el vector de cada uno de los equipos.

Para determinar si existía asociación entre las variables se obtuvieron los valores de Rho de Spearman mediante una correlación bivariada, considerando como significativos aquellos resultados con $p<0.05$, y además analizando el tamaño del efecto mediante los coeficientes de correlación.

El cálculo de ángulos, vectores y centroides ha sido realizado con las librerías de cálculo estadístico y librerías gráficas proporcionadas por $\mathrm{R}$.

El instrumento estadístico utilizado fue el paquete estadístico SPSS v.20 para Windows, y el lenguaje R en su entorno gráfico RStudio. Para la aplicación de usuario web se ha usado JS (javascript) v.1.8.5, y para el servidor se ha utilizado API RestFul hecha en Python. 


\section{Resultados}

\subsection{Latitud final del colocador}

En la Figura 8, se representa la distribución de frecuencias de la variable Latitud final del colocador ( $\mathrm{N}=2990)$, caracterizada por una fuerte acumulación de frecuencias en la zona 6 (41.4\%), seguidas por la zona 7 (23.8\%) y por la zona 5 (19.9\%).

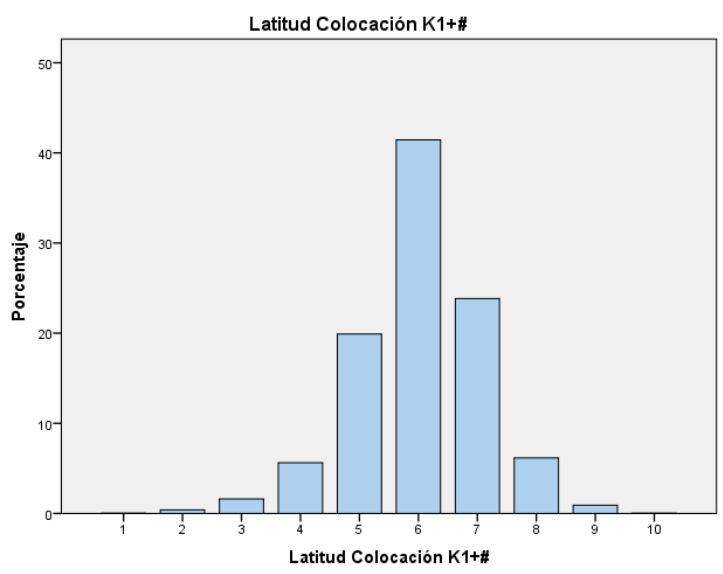

Figura 8: Frecuencias relativas de la variable Latitud final del colocador

\subsection{Profundidad final del colocador}

En la Figura 9, se representa la distribución de frecuencias de la variable Profundidad final del colocador ( $\mathrm{N}=2990)$, caracterizada por una fuerte acumulación de frecuencias en la fila $1(54.8 \%)$, seguido por la fila $2(30.9 \%)$.

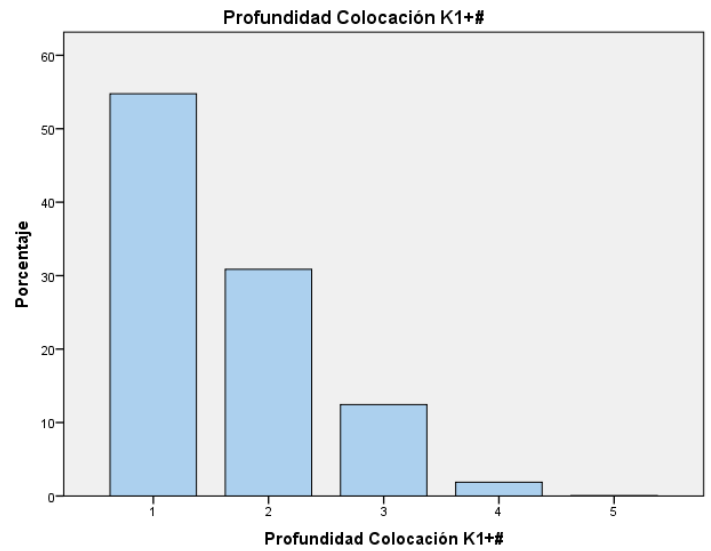

Figura 9: Frecuencias relativas de la variable Profundidad final del colocador 


\subsection{Frecuencias de todos los equipos de la posición final del colocador}

En la Figura 10, se puede observar la distribución de frecuencias absolutas y relativas de la latitud y la profundidad final del colocador, solamente para las jugadas en que en K1 haya disponibilidad del primer tiempo, de todos los equipos $(\mathrm{N}=2990)$. Podemos ver cómo la mayor frecuencia se encuentra en latitud 6 y profundidad 1 (23.5\%), de manera que conforme se va expandiendo, va disminuyendo, creando así un área.

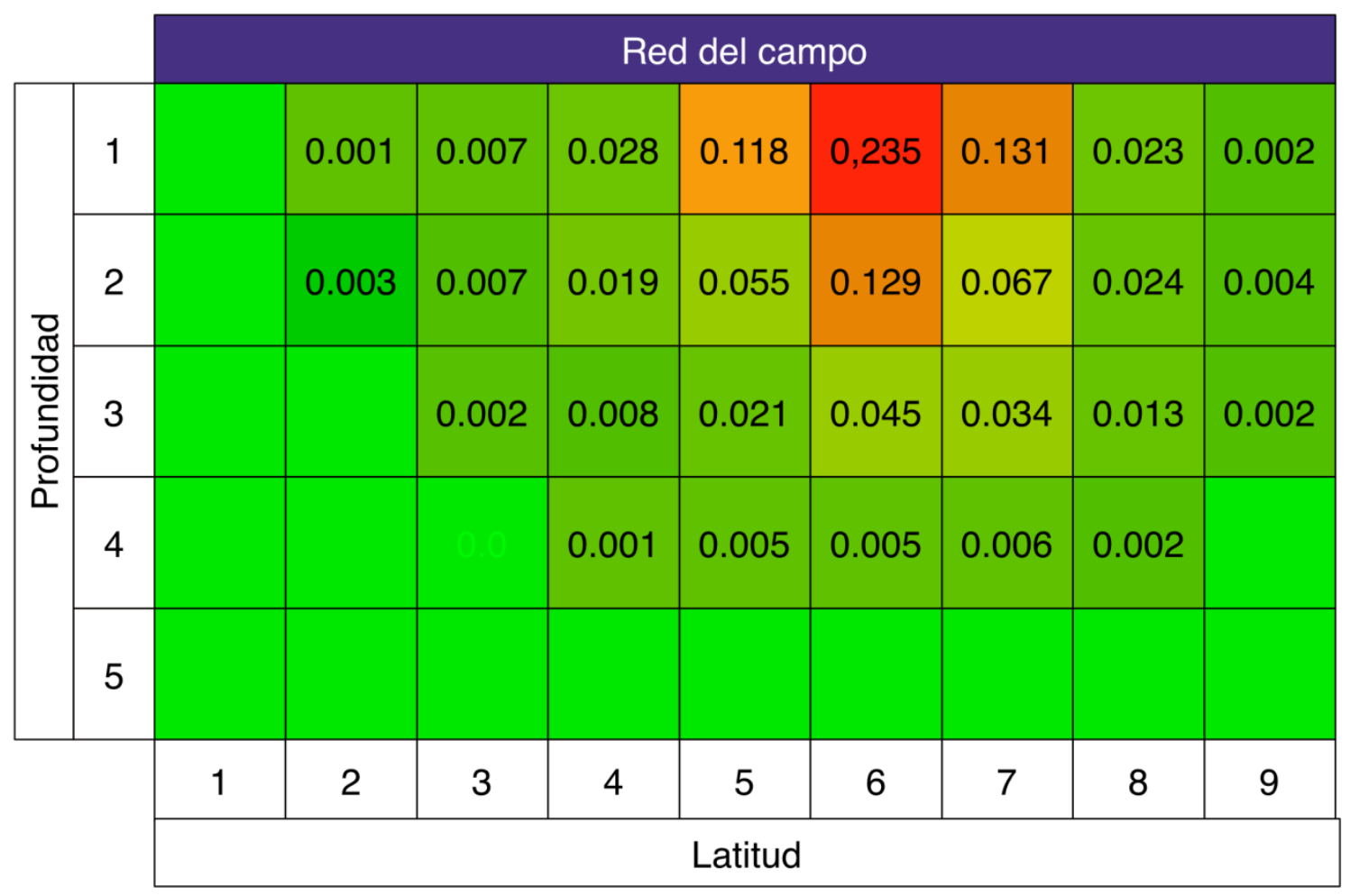

Figura 10. Frecuencias de todos los equipos de la posición final del colocador.

Se ha hecho una aplicación interactiva donde se pueden consultar las frecuencias de cada equipo de forma individual, así como el centroide, vector y ángulo. De esta manera tan gráfica, podemos incluso comparar dos equipos a la vez, entrando en la web: https://atolon.ugr.es/voleibol/\#, dentro del apartado de diagramas de densidad. 


\subsection{Vector, ángulo y centroide de cada equipo}

En la Tabla 2, se puede observar el vector, ángulo, latitud y profundidad del centroide de cada uno de los equipos, una vez calculado. Podemos ver la similitud entre los vectores, pero destaca Cuba con 1.354 y Francia es el valor más bajo con 1.058 .

Tabla 2: Vector, ángulo y centroide de cada equipo (unidades arbitrarias).

\begin{tabular}{|c|c|c|c|c|}
\hline Equipo & Vector & Ángulo & $\begin{array}{l}\text { Latitud del } \\
\text { Centroide }\end{array}$ & $\begin{array}{l}\text { Profundidad del } \\
\text { Centroide }\end{array}$ \\
\hline España & 1.121 & 0.387 & 5.923 & 1.538 \\
\hline Francia & 1.058 & 0.508 & 6.015 & 1.424 \\
\hline Rep.Checa & 1.098 & 0.501 & 6.027 & 1.463 \\
\hline Argentina & 1.189 & 0.599 & 6.171 & 1.482 \\
\hline Alemania & 1.239 & 0.368 & 5.946 & 1.656 \\
\hline Bulgaria & 1.255 & 0.35 & 5.931 & 1.679 \\
\hline USA & 1.338 & 0.404 & 6.026 & 1.73 \\
\hline Rusia & 1.306 & 0.448 & 6.066 & 1.677 \\
\hline Italia & 1.224 & 0.386 & 5.961 & 1.634 \\
\hline Serbia & 1.282 & 0.421 & 6.024 & 1.67 \\
\hline Cuba & 1.354 & 0.363 & 5.981 & 1.766 \\
\hline Brasil & 1.281 & 0.466 & 6.076 & 1.644 \\
\hline
\end{tabular}

En la Figura 11, se pueden observar los centroides de todos los equipos, así como el centroide medio, unido a la línea roja, que representa el vector procedente del centro de densidad. Se puede apreciar cómo Cuba es el equipo con mayor profundidad (1.766) y Francia el que menos (1.424). Sin embargo, Argentina es el más alejado con respecto a los demás, con la mayor latitud (6.171) y el mayor ángulo (0.599). 


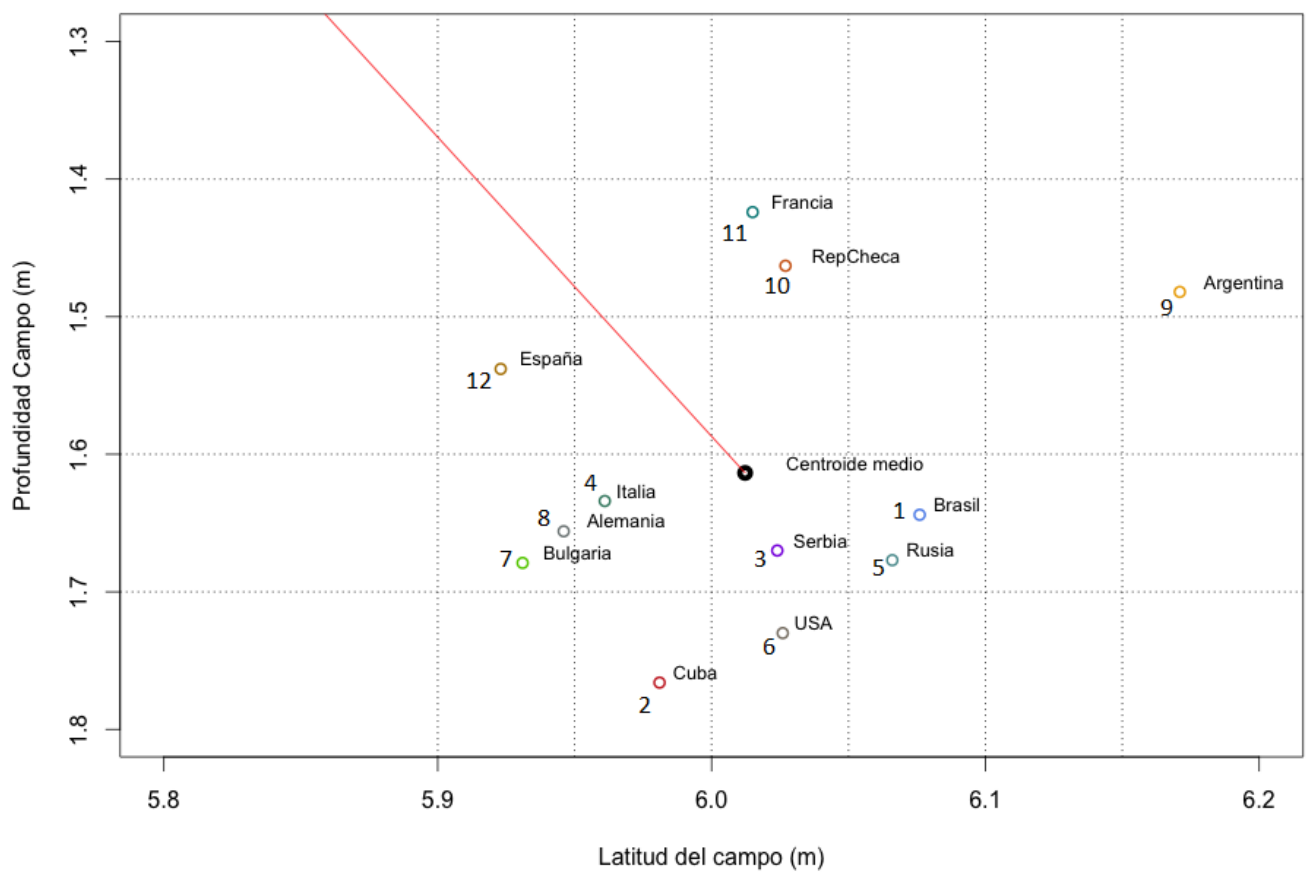

Figura 11. Centroides de todos los equipos en zona ampliada. Los números corresponden con la clasificación del campeonato del Mundo 2010 de Voleibol masculino.

\subsection{Correlación bivariada entre equipo y vector}

En la Tabla 3, se puede observar la correlación entre las variables Clasificación equipos y Vector con una significación de $p<0.01$ y un tamaño del efecto muy alto con $r_{s}=0.783$, según el coeficiente de correlación de Rho de Spearman. Respecto a la variable Ángulo, existe una correlación negativa, que aunque no da significativo ( $p>0.05$ ), tiene un tamaño del efecto levemoderado $\left(r_{s}=-0.273\right.$ y $\left.r_{s}=-0.441\right)$, según el coeficiente de correlación de Rho de Spearman.

Tabla 3. Correlación bivariada de las variables Clasificación equipos, vector y ángulo.

\begin{tabular}{|c|c|c|c|c|c|}
\hline & & & Equipo & vector & Ángulo \\
\hline \multirow{6}{*}{$\begin{array}{l}\text { Rho de } \\
\text { Spearman }\end{array}$} & \multirow{2}{*}{ Equipo } & Coeficiente de correlación & 1,000 &, $783^{* *}$ &,- 273 \\
\hline & & Sig. (bilateral) & . & ,003 & 391 \\
\hline & \multirow[t]{3}{*}{ Vector } & Coeficiente de correlación &, $783^{* *}$ & 1,000 &,- 441 \\
\hline & & Sig. (bilateral) & ,003 & & 152 \\
\hline & & Coeficiente de correlación &,- 273 &,- 441 & 1,000 \\
\hline & Ángulo & Sig. (bilateral) & ,391 & 152 & . \\
\hline
\end{tabular}




\section{Discusión}

El principal objetivo de este estudio fue analizar si el área de disponibilidad del primer tiempo de los equipos era un indicador de rendimiento, evaluando la relación que tiene ese valor en el puesto de cada equipo en la clasificación final. Los resultados sugieren, que existe relación entre el área de disponibilidad del primer tiempo y la clasificación de los equipos $\left(p<0.01 ; r_{s}=0.783\right)$, de manera que cuanto mayor sea el área disponible, mayor será la disponibilidad de ataque de primer tiempo, y por tanto, mayor será el rendimiento de un equipo. Con respecto al ángulo, aunque no haya significación ( $p>0.05)$, su tamaño del efecto leve-moderado, nos dice que los mejores equipos tienden a acumular más casos en la zona central. En la Figura 11, se puede observar cómo los equipos con una clasificación más alta, tienen sus centroides más alejados del centro de densidad, por lo que sus áreas de disponibilidad del primer tiempo son mayores. Esto le permite al equipo tener un rango mayor de espacio dónde el colocador dispone de todas las opciones de decisión, y por tanto una dependencia menor de la precisión en la recepción del saque.

Para generar un valor que nos permitiese resumir ese espectro, hemos calculado el centroide. Otros estudios en análisis de juego (Folgado et al., 2014; Frencken et al., 2011), han encontrado la utilidad de este indicador geométrico para describir la distribución de jugadores, pero como un punto dinámico, ya que varía en función de la posición del juego. Nuestra aportación, si bien es una aplicación totalmente original, refuerza el interés de este cálculo como descriptor del análisis táctico, puesto que su cálculo parte de un centro de densidad fijo, que es igual en todos los equipos.

Diversos estudios han demostrado, que el ataque es la acción que más se correlaciona con el éxito (Castro et al., 2011; Nikos y Elissavet, 2011), y más concretamente, los tiempos de ataque rápido (Asterios et al., 2009; Costa et al., 2011). Esto se debe a que este tipo de ataques crean un déficit de tiempo para el oponente, que le impide organizar su sistema defensivo de forma correcta (Afonso et al., 2010; Asterios et al., 2009). Teniendo en cuenta que el colocador es el jugador que distribuye el juego, y su relación directa con el éxito (Buscà y Febrer, 2012; Morales, 2011; Palao et al., 2005; Papadimitriou et al., 2004; Ureña, 1998), cabe destacar la importancia de que el colocador disponga de todas las opciones de ataque. Así pues, siguiendo a Afonso et al. (2010), la disponibilidad del atacante central para ataques de primer tiempo es necesario para que el ataque sea ágil y ofensivo, siempre y cuando exista una adecuada relación espacial entre colocador, central y balón, así como una coordinación 
temporal de ambos jugadores. De nuestros resultados se desprende, que esa relación espacial varía en función del equipo y de su área de disponibilidad del primer tiempo, por lo que se puede considerar como un indicador de rendimiento.

Numerosos estudios (Barzouka et al., 2006; Nikos y Elissavet, 2011; Zetou et al., 2007), han tratado la influencia que tiene la calidad de la recepción en el éxito de las acciones posteriores del juego. Algunos autores (Costa et al., 2011; Moras et al., 2008; Zetou et al., 2007) han determinado la calidad de la recepción delimitando un espacio donde llegaba el balón y valorando en función de su precisión, siguiendo los criterios del Data Volley System Valuation. No obstante, nuestros resultados nos permiten cuestionar si la calidad en recepción, medida desde la precisión, puede resultar un indicador insuficiente. Los resultados han demostrado el carácter variable de la disponibilidad del ataque de primer tiempo y su influencia en la clasificación del equipo. Esto afecta al valor absoluto que la investigación hasta ahora ha concedido a la calidad de la recepción como indicador de rendimiento de ataque.

\section{Conclusiones}

El centroide es un valor resumen del área de disponibilidad del primer tiempo, que nos permite comparar unos equipos con otros, a partir de la distribución de frecuencias de colocación a lo largo de la superficie de juego en las que el equipo mantiene esa disponibilidad.

El área de disponibilidad del primer tiempo de los equipos es un indicador de rendimiento, en relación con el puesto de cada equipo en la clasificación final.

La precisión en la recepción como indicador del rendimiento, está limitado por la capacidad mayor de algunos equipos de mantener, desde recepciones menos precisas, la disponibilidad del ataque del primer tiempo. 


\section{Aplicaciones y futuras líneas de investigación}

Los resultados del estudio muestran que, cuanto mayor sea la calidad del equipo, el área en la que el primer tiempo puede atacar es también mayor, aunque el colocador esté más separado. Por lo tanto, el bloqueo rival no debe confiarse en que no se va a atacar primer tiempo, y tenerlo en cuenta para establecer una estrategia de juego. Partiendo de esta idea, sería interesante que los equipos entrenen con sus centrales y colocador, la capacidad de mantener la disponibilidad del primer tiempo en situaciones mucho más diversas, independientemente de que el colocador esté en la zona ideal o no. De cara a las decisiones de selección de jugadores y de objetivos de entrenamiento, aquí encontramos dos factores que se contrabalancean y permiten una mayor información a la hora de tomar decisiones: la precisión en la recepción del saque y la capacidad de mantener disponible el primer tiempo de ataque.

Una de las limitaciones de este estudio es que sólo se ha realizado con equipos de un campeonato, por lo que en futuros estudios sería interesante calcular los centroides de los mismos equipos y de otros en diferentes campeonatos, para determinar si la correlación de clasificación de los equipos y área disponible de primer tiempo se mantiene en el tiempo. Asimismo, estudiar los centroides de equipos femeninos y de otras categorías, para poder comparar las diferencias entre ellos y con respecto a la élite.

Por otra parte, el desarrollo de la aplicación interactiva, la cual se puede ver en https://atolon.ugr.es/voleibol/\#, es una herramienta muy útil tanto para jugadores como para entrenadores, porque permite ver de forma gráfica la distribución de frecuencias del colocador, y el área de disponibilidad del primer tiempo, por lo que en función de eso, pueden establecer el plan de juego.

Estamos trabajando en futuros estudios que establecen relación entre las frecuencias de distribución del colocador, el efecto que tiene el colocador sobre el bloqueo contrario y la eficacia del ataque, buscando análisis comparativos y con aplicaciones interactivas que le den flexibilidad al análisis. 


\section{Agradecimientos}

Al Ministerio de Ciencia e Innovación, por la financiación del proyecto Sistema MasVb de Evaluación Competitiva y Orientación Técnica para la Superliga Española de Voleibol (DEP201127503).

A mi grupo de investigación por guiarme en el proceso siempre con una sonrisa, y en especial a Marisa, por ser mi mano derecha en el camino del aprendizaje.

A Manu y Benítez por sus aportaciones con la estadística y diseño de la página web.

A Aurelio, por su apoyo y confianza en mí, guiándome y motivándome desde el principio hasta el final. 


\section{Referencias}

Afonso, J., Mesquita, I., Marcelino, R. y Silva, J. (2010). Analysis of the setter's tactical action in high-performance women's Volleyball. Kinesiology, 42(1), 82-89.

Afonso, J. y Mesquita, I. (2011). Determinants of block cohesiveness and attack efficacy in high level women's volleyball. European Journal of Sport Science, 11(1), 69-75.

Asterios, P., Kostantinos, C., Athanasios, M. y Dimitrios, K. (2009). Comparison of technical skills effectiveness of men's National Volleyball teams. International Journal of Performance Analysis in Sport, 9(1), 1-7.

Barzouka, K., Nikolaidou, M. E., Malousaris, G. y Bergeles, N. (2006). Performance excellence of male setters and attackers in complex I and II on volleyball teams in the 2004 Olympic Games. International Journal of Volleyball Research, 9(1), 19-24.

Beal D. (1992). Combinaciones de Ataque. EnBertucci, B. (Ed.),Guía de Voleibol de la Asociación de Entrenadores Americanos de Voleibol. Barcelona. Paidotribo.

Buscà, B. y Febrer, J. (2012), Temporal fight between the middle blocker and the setter in high level volleyball, International Journal of Medicine and Science of Physical Activity and Sport, 12(46), 313-327.

Castro, J. y Mesquita, I. (2008), Implications of offensive spacing in elite male volleyball attack characteristics, Portuguese Journal of Sport Sciences, 8(1), 114-125.

Castro, J., Souza, A., y Mesquita, I. (2011). Attack efficacy in volleyball: Elite male teams. Perceptual and Motor Skills, 113, 395-408.

Cohen, J. (1960). A coefficient of agreement for nominal scales. Educational and psychological measurement, 20(1), 37-46.

Costa, G., Mesquita, I., Greco, P. J., Ferreira, N. N. y Morales, J. C. (2011). Relação saque, recepção e ataque no voleibol juvenil masculino. Motriz, Rio Claro, 17(1), 11-18. 
Davids, K. Glazier, P. Arau'jo, D. y Bartlett, R. (2003). Movement systems as a dynamical systems: The funtional role of variability and ist implications for sports medicine. Sport Med 33: 245-260.

Fleiss, J. (2003). Statistical methods for rates and proportions.3rd ed. Wiley-Interscience.

Garganta, J. (2009). Trends of tactical performance analysis in team sports: bridging the gap between research, training and competition. Revista Portuguesa de Ciências do Desporto, 9(1), 81-89.

Jager, J. y Schollhorn, W. (2007). Situation-oriented recognition of tactical patterns in volleyball. Journal of Sports Sciences, 25(12), 1345-1353.

Nikos, B. y N. M. Elissavet (2011). "Setter's performance and attack tempo as determinants of attack efficacy in Olympic-level male volleyball teams." International Journal of Performance Analysis in Sport, 11(3): 535-544.

Mapelli, A., Zago, M., Fusini, L., Galante, D., Colombo, A. y Sforza, C. (2014). Validation of a protocol for the estimation of three-dimensional body center of mass kinematics in sport. Gait y Posture, 39(1), 460-465. doi:10.1016/j.gaitpost.2013.08.025

Morales, S. C. (2011). Variables significativamente influyentes en el rendimiento del pasador de voleibol. Revista Internacional de Medicina y Ciencias de la Actividad Física y del Deporte/International Journal of Medicine and Science of Physical Activity and Sport, 11(42), 347-361.

Moras, G., Buscà, B., Peña, J., Rodríguez, S., Vallejo, L., Tous-Fajardo y J., Mujika, I. (2008). A comparative study between serve mode and speed and its effectiveness in a high-level volleyball tournament. Journal of Sports Medicine and Physical Fitness; 48, 1; ProQuest pg. 31

Palao, J., Santos, J. y Ureña, A. (2005), The effect of setter's position on the spike in volleyball, Journal of Human Movement Studies, 48(1), 25-40. 
Papadimitriou, K., Pashali, E., Sermaki, I., Mellas, S., y Papas, M. (2004). The effect of the opponents' serve on the offensive actions of Greek setters in volleyball games. International Journal of Performance Analysis in Sport, 4(1), 23-33.

Queiroga, M., Matias, C., Greco, P., Graça, A. and Mesquita, I. (2005). The dimension of the high-level setter's tactical strategic knowledge: Study with setters of Brazilian national teams. Brazilian Journal of Physical Education, Special Edition, 111-119.

Tabachnick, B. y Fidell, L. (2013). Using multivariate statistics (6th ed.). Boston: Allyny Bacon.

Ureña, A. (1998). La incidencia de la misión ofensiva en el rendimiento de la recepción del saque en voleibol (Tesis Inédita de doctorado). Departamento de personalidad, evaluación y tratamiento psicológico. Universidad de Granada.

Zetou, E., Moustakidis, A., Tsigilis, N. y Komninakidou, A. (2007). Does effectiveness of skill in Complex I predict win in Men's Olympic Volleyball Games? Journal of Quantitative Analysis in Sports, 3(4), 1-9. 